\title{
Antibacterial Envelope Use for the Prevention of Surgical Site Infection in Spinal Cord Stimulator Implantation Surgery: A Retrospective Review of 52 Cases
}

Jonathan M Hagedorn (D)

Nicholas Canzanello (D)

Markus A Bendel (DD

Thomas P Pittelkow

Tim J Lamer (iD)

Department of Anesthesiology and Perioperative Medicine, Division of Pain Medicine, Mayo Clinic, Rochester, MN, 55905, USA
Correspondence: Jonathan M Hagedorn Department of Anesthesiology and Perioperative Medicine, Division of Pain Medicine, Mayo Clinic, Rochester, MN, 55905, USA

Fax +I 507-266-7732

Email jonhagedornmd@yahoo.com
Objective: Surgical site infection in patients who undergo spinal cord stimulator implant surgery represents a significant concern in terms of increased health care costs and patient morbidity. The use of antibacterial envelopes in spinal cord stimulator implant surgeries has not been previously described. The aim of this retrospective review was to evaluate the effectiveness of the antibacterial envelope in reducing surgical site infection in spinal cord stimulator implant surgeries when used adjunctively to standard infection prevention measures.

Materials and Methods: The study included 52 patients, all of whom were implanted with a spinal cord stimulator between January 2015 and November 2020. To be included, patients were required to have had an antibacterial envelope utilized at the time of surgery. Patient records were retrospectively reviewed. All patients who received an antibacterial envelope at the time of implant surgery were included.

Results: Data was collected and analyzed on 52 permanent SCS implantations, including primary implantation $(n=26)$ and revision surgery $(n=26)$. All patients were at least three months post-operative from the implant surgery (average follow-up time period was 518.4 days). There were no surgical site infections reported in the 52 patient cohort.

Conclusion: Antibiotic impregnated envelopes appear to be a safe and effective modality to decrease surgical site infection risk in spinal cord stimulation implant surgeries.

Keywords: spinal cord stimulation, surgical site infection, outcomes, infection prevention

\section{Introduction}

The use of spinal cord stimulation (SCS) for the treatment of intractable pain has increased significantly in the past decade. This in large part has been driven by technological advancements and research outcomes supporting expanded indications, and increased access to advanced pain therapies. However, newer technologies are still plagued by similar complications as earlier devices. This includes consequential and expensive post-operative outcome, surgical site infection (SSI).

Prior large retrospective studies have reported SCS infection rates ranging from 2.5 to $6 \%{ }^{1,2}$ Not only does this lead to increased healthcare expenditures by greater than $\$ 60,000$ per SSI event, it often leads to device explant, therapy discontinuation, and decreased patient quality of life. ${ }^{3}$ In fact, only $26 \%$ of patients will be reimplanted after SCS explant for SSI. ${ }^{3}$ While the Neurostimulation Appropriateness 
Consensus Committee (NACC) guidelines, US Centers for Disease Control and Prevention (CDC), United Kingdom National Institute for Health and Care Excellence (NICE), and Surgical Care Improvement Project (SCIP) have all defined infection control recommendations, physician compliance remains less than ideal and SSI rates have not changed significantly with time. ${ }^{4-10}$ However, this does not change the overriding goal: to achieve optimal patient care and overall healthcare stewardship, the field of neuromodulation must continue to strive for lower infection rates and find methods that are easily adoptable into a variety of clinical settings.

A single-use, absorbable, multifilament mesh antibacterial envelope (TYRX Absorbable Antibacterial Envelope, Medtronic, Fridley, MN, USA) is available for SCS implant surgical procedures. The implantable pulse generator (IPG) is placed within the antibiotic envelope which is then inserted in the IPG pocket site. Previous studies have shown that Staphylococcus aureus is the most common infectious agent and the IPG pocket is the most common site of infection. ${ }^{11}$ Often, the IPG is small enough to allow for the antibiotic pouch to be trimmed to size. The remnant pouch pieces can be placed in the lead anchoring incision so that all implantation incisions receive antibiotic coverage, though it is important to note that this is an offlabel use of the product. The envelope is impregnated with minocycline and rifampin, and elutes these antibiotics for a minimum of seven days after implantation. By approximately nine weeks post-implantation, the envelope is fully absorbed.

In patients with an elevated risk of SSI, as deemed by the implanting physician after a thorough medical history and physical exam, it is our practice to consider utilizing an antibacterial envelope. Common scenarios that may constitute use of an antibacterial envelope include: current cigarette smoker, history of diabetes mellitus, history of prior surgical site infection, revision surgery, or use of immunosuppressive medications. A librarian-assisted literature search of the Ovid MEDLINE, Embase, and PubMed databases was performed and did not identify any published full manuscripts on this topic. To our knowledge, the use of antibacterial envelopes has not been previously reported in the SCS literature. The aim of this retrospective review was to evaluate the effectiveness of an antibacterial envelope on SSI in SCS implant surgeries (including primary implant and revision surgeries) when used adjunctively to standard infection prevention measures (Table 1).

\section{Methods}

Permission to conduct this study was granted by Mayo Clinic's Institutional Review Board, who deemed it an exempt protocol given the retrospective study design and waived the need for consent. The guidelines in the Declaration of Helsinki were followed. All patients' records were retrospectively reviewed and recorded data

Table I Standard Infection Prevention Measures

\begin{tabular}{|l|l|}
\hline Preoperative & Standard Infection Prevention Measures \\
\hline & $\begin{array}{l}\text { Preoperative glycemic control (in patients with history of diabetes mellitus, require HbAIc < 8\%) } \\
\text { MSSA/MRSA nasal swab screening; mupirocin nasal ointment for MRSA+ patients } \\
\text { Preoperative shower the night before and morning of surgery } \\
\text { Chlorhexidine scrub the night before and morning of surgery }\end{array}$ \\
\hline Intraoperative & $\begin{array}{l}\text { Patient skin cleansing with chlorhexidine (povidone-iodine in chlorhexidine-allergic patients) } \\
\text { Weight-based antibiotic dosing } \\
\text { Antimicrobial incise drape } \\
\text { Double glove throughout surgery } \\
\text { Surgical hemostasis } \\
\text { Wound irrigation with normal saline, antibacterial-based solution, or iodine-based solution } \\
\text { Apply sterile occlusive dressing }\end{array}$ \\
\hline Postoperative & $\begin{array}{l}\text { Oral antibiotics } \\
\text { Sterile occlusive dressing remains in place for 48-72 hours; patient may remove dressing and resume showering after this time period } \\
\text { No submerging of incisions under water until post-operative clinic visit }\end{array}$ \\
\hline
\end{tabular}

Abbreviations: HbAIC, hemoglobin AIC; MSSA, methicillin-sensitive Staphylococcus aureus; MRSA, methicillin-resistant Staphylococcus aureus. 
was protected on a secure database. The study included 52 patients, all of whom were implanted with a spinal cord stimulator device between January 2015 and November 2020. To be included, patients were required to have had an antibacterial envelope utilized at the time of surgery. All SCS implants that utilized an antibacterial envelope at the time of surgery were included in the study. The decision to use an antibacterial envelope was at the surgeon's discretion. At our institution, antibacterial envelopes are typically used in surgical cases deemed to be at an increased risk of post-operative infection, often due to medical comorbidities, history of prior SSI, or revision surgery. Demographic data, including age, gender, body mass index (BMI), history of diabetes, history of tobacco use, and history of prior neuromodulation SSI was collected. Surgical details, including date of implant, primary versus revision surgery designation, trial length, trial type (percutaneous versus staged), choice of intra-operative antibiotic, and whether post-operative antibiotics were prescribed were reviewed. The date of the last follow-up visit was used to calculate follow-up time periods.

Surveillance for SSI involved scheduled in-office follow-up visits at one day, ten days, six weeks, and three months post-operative. Additionally, patients are seen inoffice as needed for device reprogramming or treatment of other chronic pain conditions, and the surgical sites are visualized at those times to ensure a normal appearance. Lastly, during the retrospective data collection, the electronic medical records were reviewed for mention of device infection.

\section{Results}

Data was collected and analyzed on 52 permanent SCS implantations (primary implantation: $\mathrm{n}=26$ [50\%], revision surgery: $\mathrm{n}=26[50 \%])$. The average age was 62.2 years. There were 27 females (51.9\%) and 25 males (48.1\%). The average follow-up time period was 518.4 days (1.4 years). All patients included in the analysis were at least three months post-operative from the implant surgery. There were no SSI reported in the 52 patient cohort.

Patient-related risk factors were collected (Table 2). The average BMI was 30.5 (SD 7.8). Twenty-seven patients $(51.9 \%)$ had a BMI above 30 . Ten patients $(19.2 \%)$ had diabetes. There were six current tobacco smokers (11.5\%) and 18 former tobacco smokers (34.6\%).

Surgical-related risk factors were also collected (Table 3). All surgeries occurred at an academic medical center. There were 26 primary implants $(50 \%)$ and 26
Table 2 Patient Demographics and Risk Factors

\begin{tabular}{|l|c|}
\hline Variable & Number Reported \\
\hline Total Implants & 52 \\
\hline Age (SD) & $62.2(16.6)$ \\
\hline Gender & 25 \\
Male & 27 \\
Female & \\
\hline BMI & $30.5(7.8)$ \\
Mean (SD) & 25 \\
$\leq 30$ & 27 \\
$>30$ & \\
\hline Diabetes & 10 \\
Yes & 42 \\
No & \\
\hline Tobacco use & 6 \\
Yes & 28 \\
No & 18 \\
Former & \\
\hline
\end{tabular}

Abbreviations: SD, standard deviation; BMl, body mass index.

revision surgeries $(50 \%)$. All implants and revisions involved cylindrical leads. For the primary implants specifically, 4 patients had a trial length of 5 days or shorter, while 22 patients had a trial length longer than 5 days. There were 20 percutaneous trials and 6 staged trials. In the revision surgeries, 14 were IPG exchanges, 1 was a lead-only revision, and 11 were combined IPG and lead revisions. Weight-based intra-operative antibiotics were given appropriately for every case. Fourteen patients were found to be colonized with Staphylococcus aureus pre-operatively via nasal swab, were prescribed nasal mupirocin ointment pre-operatively, and were given Vancomycin intra-operatively. A sterile occlusive dressing was applied for every case. Post-operative antibiotics were prescribed in 41 cases (78.8\%) for a mean of 6 days.

There were no surgical complications encountered from the use of the antibacterial envelope, including delayed or interrupted wound healing, skin irritation, allergic reactions, seroma or hematoma formation, or increased post-operative pain.

\section{Discussion}

One of the most serious complications associated with SCS implant surgery is SSI. Associated morbidity and mortality secondary to SSI results in numerous downstream consequences for the patient and the general safety of SCS surgery. The results presented in this retrospective 
Table 3 Surgical and Post-Operative Factors

\begin{tabular}{|c|c|}
\hline Variable & Number Reported \\
\hline Total Implants & 52 \\
\hline \multicolumn{2}{|l|}{ Revision Surgery } \\
\hline Yes & 26 \\
\hline No & 26 \\
\hline \multicolumn{2}{|l|}{ Revision Surgery Details } \\
\hline IPG only & 14 \\
\hline Leads only & 1 \\
\hline IPG and Leads & 11 \\
\hline \multicolumn{2}{|l|}{ Trial length } \\
\hline Mean & $7(2.3)$ \\
\hline$\leq 5$ days & 4 \\
\hline$>5$ days & 22 \\
\hline \multicolumn{2}{|l|}{ Trial type } \\
\hline Percutaneous & 20 \\
\hline Staged & 6 \\
\hline \multicolumn{2}{|l|}{ Institutional Setting } \\
\hline Academic & 52 \\
\hline \multicolumn{2}{|l|}{ Intraoperative antibiotics } \\
\hline Cefazolin & 28 \\
\hline Vancomycin & 14 \\
\hline Clindamycin & 7 \\
\hline Cefepime & 1 \\
\hline Vancomycin and Cefazolin & 1 \\
\hline Vancomycin and Cefepime & 1 \\
\hline \multicolumn{2}{|l|}{ Occlusive dressing } \\
\hline Yes & 52 \\
\hline \multicolumn{2}{|l|}{ Post-operative antibiotics } \\
\hline Yes & 41 \\
\hline No & 11 \\
\hline \multicolumn{2}{|l|}{ Post-operative antibiotics timing } \\
\hline Mean (SD) & $6(2.5)$ \\
\hline I day & 5 \\
\hline > I day & 36 \\
\hline
\end{tabular}

Abbreviations: IPG, implantable pulse generator; SD, standard deviation.

review suggest that use of an absorbable antibacterial envelope may be effective in reducing the risk of SSI in primary and revision SCS implant surgeries. Additionally, careful assessment of comorbid perioperative risk factors may play a crucial role when establishing interventions for infection prevention.

Prior large retrospective studies have found higher risk of SSI with standard prophylactic measures than we found in the reported patient population with use of standard prophylactic measures along with an antibacterial envelope. ${ }^{1,12}$ Hoelzer et al performed a multisite retrospective review and analyzed 2737 permanent SCS implants. ${ }^{1}$ Surgical site infection was reported in 67 of those cases $(2.45 \%)$. They found statistically significant higher rates of infection when implants were performed at academic centers $(\mathrm{p}=0.03)$, when trials lasted longer than five days $(\mathrm{p}=0.02)$, in percutaneous trials versus staged trials $(p=0.05)$, when an occlusive dressing was not applied $(\mathrm{p}<0.001)$, and when post-operative antibiotics were not prescribed $(p=0.001)$. When primary implants were compared to revision surgery, there was a trend toward higher infection risk with revision surgeries developing SSI (3.09\%) versus primary implants $(2.19 \%$; $\mathrm{p}=$ 0.17). When we compare these published risk factors to the patient cohort in the current manuscript, a number of common higher-risk scenarios were noted. In the data presented, all surgeries were performed at an academic center with trainee involvement. The majority of trials were percutaneous trials $(20 / 26,76.9 \%)$ versus staged trials. The majority of trials lasted longer than five days $(22 / 26,84.6 \%)$ with a mean of seven days. Lastly, postoperative antibiotics were not given in eleven cases $(11 / 52$, $21.2 \%$ ). However, even with these potential risk factors, not a single SSI was identified when an antibacterial envelope was used at the time of surgery. These results add to the novel body of literature supporting the appropriate use and efficacy of antibacterial envelope in reducing risk of SCS-associated SSI.

Falowski et al performed a review of the Truven MarketScan ${ }^{\circledR}$ databases for patients implanted with SCS between 2009-2014. ${ }^{12}$ The review included 6615 patients and 12 -month SSI rate was $3.11 \%$. Interestingly, their results showed increased age was protective against SSI. Not surprisingly, a history of infection within 12 months of SCS implant increased the risk of SSI. Similar to the Hoelzer et al study, patient BMI, history of diabetes, and current tobacco use did not result in an elevated rate of SSI. When compared to the patient population reported in this study, two patients with a history of prior SCS explant surgery within the previous 12 months due to SSI had reimplant surgery utilizing all the standard perioperative precautions plus the use of an antibacterial envelope and did not develop SSI (both patients are $>12$ months postreimplant surgery). Careful attention is required to identify and optimize perioperative risk factors. The use of an antibacterial envelope could be the cornerstone of infection prevention in this higher risk population. 
To our knowledge, no studies have specifically detailed the use of antibacterial envelope in the SCS surgical literature. However, the benefit of antibacterial envelopes has been well studied in the cardiac implantable electronic device (CIED) literature. Tarakji et al conducted a randomized controlled trial to assess the safety and efficacy of antibiotic-eluting envelopes in reducing the incidence of SSI within 12 months of CIED implantations. ${ }^{13}$ Patients were randomized in a $1: 1$ ratio to receive the envelope or not. Other standard of care measures were utilized in all patients. A total of 6983 patients were randomized to the study (3495 envelope versus 3488 control). Surgical site infection occurred in 25 patients in the envelope group $(25 / 3495$, or $0.7 \%)$ versus 42 patients in the control group $(42 / 3488$, or $1.2 \%)$ at 12 months post-implantation. Patients were then followed beyond the 12 month study period for an average follow-up of 20.7 months. Throughout the entire follow-up time period, 32 patients in the envelope group $(32 / 3495$, or $0.9 \%)$ versus 51 patients in the control group $(51 / 3488$, or $1.5 \%)$ developed a CIED-related SSI. The authors concluded that adjunctive use of an antibacterial envelope resulted in a significantly lower incidence of CIED SSI than typical practices alone. They also reported that there was no higher incidence of complications with use of the envelope.

A weakness of our manuscript is the retrospective nature of the data collection leading to an inherent inability to control for other infection mitigation practices which may have impacted the observed outcomes. This study does contain a varying range of physician implanter experience and operative techniques which can also have an effect on SSI risk - but this heterogeneity, along with including all patients treated with an antibacterial envelope in the dataset, may make the observed data more universally applicable. Despite the inclusion of many patients with clinical profiles believed to be higher risk for SSI, the sample size may not be large enough to make definitive statements. A larger prospective multisite study may be helpful in further reporting and clarifying the benefits of antibiotic envelope utilization in the SCS population. Lastly, the absence of a control group precludes determination of whether an antibacterial envelope reduces SSI risk compared to no antibacterial envelope. However, given prior reports of SSI in the $2.5-6 \%$ range, we would have expected to experience at least one SSI within this patient cohort using standard infection prevention measures.

\section{Conclusion}

Implantation of SCS therapies is rising and the surgical volume is projected to continue on an upward trajectory as new indications and technologies emerge. Understanding and mitigating the surgical complications, such as SSI, to the maximal degree possible is essential for the continued success of the field. Antibiotic impregnated envelopes appear to be a safe and effective modality to decrease SSI in the SCS implantation population.

\section{Author Contributions}

JMH and TJL were involved with study inception. JMH and NC were involved with data collection. All authors contributed to data analysis, drafting or revising the article, gave final approval of the version to be published, agreed to the submitted journal, and agree to be accountable for all aspects of the work.

\section{Funding}

There is no funding to report.

\section{Disclosure}

JMH is a consultant for Abbott, Boston Scientific, and Nevro. He serves on advisory boards for Abbott, Boston Scientific, and Nevro. MAB has funded research with Nevro. All other authors report no conflicts of interest.

\section{References}

1. Hoelzer BC, Bendel MA, Deer TR, et al. Spinal cord stimulator implant infection rates and risk factors: a multicenter retrospective review. Neuromodulation. 2017;20(6):558-562. doi:10.1111/ner.12609

2. Taylor RS, Van Buyten JP, Buchser E. Spinal cord stimulation for chronic back and leg pain and failed back surgery syndrome: a systematic review of the clinical and analysis of prognostic factors. Spine. 2005;30(1):152-160. doi:10.1097/01.brs.0000149199.68381.fe

3. Provenzano DA, Falowski SM, Xia Y, Doth AH. Spinal cord stimulation infection rate and incremental annual expenditures: results from a United States payer database. Neuromodulation. 2019;22 (3):302-310. doi:10.1111/ner.12939

4. Provenzano DA, Deer T, Luginbuhl Phelps A, et al. An international survey to understand infection control practices for spinal cord stimulation. Neuromodulation. 2016;19(1):71-84. doi:10.1111/ ner. 12356

5. Deer TR, Abd-Elsayed A, Falowski S, et al. Practice choices in targeted intrathecal drug delivery: an online surgery conducted by the polyanalgesic consensus committee. Neuromodulation. 2021.

6. Deer TR, Provenzano DA, Hanes M, et al. The neurostimulation appropriateness consensus committee (NACC) recommendations for infection prevention and management. Neuromodulation. 2017;20 (1):31-50. doi:10.1111/ner.12565

7. Berrios-Torres SI, Umscheid CA, Bratzler DW, et al. Centers for disease control and prevention guidelines for the prevention of surgical site infection, 2017. JAMA Surg. 2017;152(8):784-791. doi:10.1001/ jamasurg.2017.0904 
8. National Institute for Health and Care Excellence (NICE). Surgical site infections: prevention and treatment (NG125); April 11, 2019. Available from: https://www.nice.org.uk/guidance/ng125/resources/ surgical-site-infections-prevention-and-treatment-pdf -66141660564421. Accessed July 14, 2021.

9. Bratzler DW. The surgical infection prevention and surgical care improvement projects: promises and pitfalls. Am Surg. 2006;72 (11):1010-1016. doi:10.1177/000313480607201106

10. Bratzler DW, Hunt DR. The surgical infection prevention and surgical care improvement projects: national initiatives to improve outcomes for patients having surgery. Clin Infect Dis. 2006;43 (3):322-330. doi:10.1086/505220
11. Bendel MA, O'Brien T, Hoelzer BC, et al. Spinal cord stimulator related infections: findings from a multicenter retrospective analysis of 2737 implants. Neuromodulation. 2017;20(6):553-557. doi:10.1111/ner.12636

12. Falowski SM, Provenzano DA, Xia Y, Doth AH. Spinal cord stimulation rate and risk factors: results from a United States payer database. Neuromodulation. 2019;22(2):179-189. doi:10.1111/ ner. 12843

13. Tarakji KG, Mittal S, Kennergren C, et al. Antibacterial envelope to prevent cardiac implantable device infection. $N$ Engl $J$ Med. 2019;380(20):1895-1905. doi:10.1056/NEJMoa1901111

\section{Publish your work in this journal}

The Journal of Pain Research is an international, peer reviewed, open access, online journal that welcomes laboratory and clinical findings in the fields of pain research and the prevention and management of pain. Original research, reviews, symposium reports, hypothesis formation and commentaries are all considered for publication. The manuscript management system is completely online and includes a very quick and fair peer-review system, which is all easy to use. Visit http:// www.dovepress.com/testimonials.php to read real quotes from published authors. 